\title{
The decomposition of the loop space of the mod 2 Moore space
}

\author{
JELENA GRBiĆ \\ PAUL SELICK \\ JIE WU
}

\begin{abstract}
In 1979 Cohen, Moore and Neisendorfer determined the decomposition into indecomposable pieces, up to homotopy, of the loop space on the mod $p$ Moore space for primes $p>2$ and used the results to find the best possible exponent for the homotopy groups of spheres and for Moore spaces at such primes. The corresponding problems for $p=2$ are still open. In this paper we reduce to algebra the determination of the base indecomposable factor in the decomposition of the mod 2 Moore space. The algebraic problems involved in determining detailed information about this factor are formidable, related to deep unsolved problems in the modular representation theory of the symmetric groups. Our decomposition has not led (thus far) to a proof of the conjectured existence of an exponent for the homotopy groups of the mod 2 Moore space or to an improvement in the known bounds for the exponent of the 2-torsion in the homotopy groups of spheres.
\end{abstract}

$55 \mathrm{P} 35 ; 16 \mathrm{~W} 30$

\section{Introduction}

In a series of celebrated papers [4; 3; 5] dating from 1979, Cohen, Moore and Neisendorfer determined for primes $p>3$ the decomposition of the loop space of the mod $p$ Moore space $\Omega P^{m}\left(p^{r}\right)$ into factors which are indecomposable up to homotopy equivalence. By this method they found elements of order $p^{r+1}$ in the homotopy groups of $\Omega P^{m}\left(p^{r}\right)$ and showed that $p^{r+1}$ is an exponent for these homotopy groups. That is, they showed that these groups contain no elements of order greater than $p^{r+1}$ or equivalently that they are annihilated by multiplication by $p^{r+1}$. This fits with a conjecture of Barratt which says that if the identity map of a double suspension has order $p^{r}$ then its homotopy groups should have exponent $p^{r+1}$. As a very important application [3], they also used their decomposition of $\Omega P^{2 n+1}\left(p^{r}\right)$ to construct a map $\pi: \Omega^{2} S^{2 n+1} \longrightarrow S^{2 n-1}$ having the property that the composite $E^{2} \circ \pi: \Omega^{2} S^{2 n+1} \longrightarrow \Omega^{2} S^{2 n+1}$ with the double suspension $E^{2}$ is multiplication 
by $p$, which has (by induction) the corollary that the $p$-torsion of $\pi_{k}\left(S^{2 n+1}\right)$ has exponent $p^{n}$ for all $k$.

The same decomposition and corollary for the prime 3 were obtained shortly afterward by Neisendorfer [7] by working around the technical difficulties created by the failure of the Jacobi identity to hold for the Samelson product of mod 3 homotopy. The corresponding problems for the prime 2 are of greater difficulty, and many questions remain open. For $r>1$, the decomposition of the looped Moore space $\Omega P^{2 n+1}\left(2^{r}\right)$ into indecomposable pieces (up to homotopy) was eventually obtained by Cohen [2]. By this means he was able to show that these Moore spaces have a homotopy exponent (later improved by Theriault [14] to the best possible exponent when $r \geq 6$ ). Unfortunately the decomposition of $\Omega P^{2 n+1}$ (4) gave no improvement on the exponent of the $2-$ torsion of $\pi_{*}\left(S^{2 n+1}\right)$ which was already known to have exponent $4^{n}$ from work of James [6] in 1955. This exponent was improved to approximately $2^{3 n / 2}$ by the second author [8], but no elements of order greater than $2^{n+\epsilon}$ (where $\epsilon=0$ or 1 depending upon the parity of $n$ ) are known in the 2-torsion of the homotopy groups of $S^{2 n+1}$, and this is the conjectured exponent. It is still unknown whether the Moore space $P^{2 n+1}(2)$ has any exponent at all. Since the identity map of $P^{2 n+1}(2)$ has order 4, according to Barratt's conjecture it ought to have exponent 8 . At this stage, it is clear that a more detailed study of the mod 2 Moore space and homotopy decompositions of $\Omega P^{k}(2)$ is needed.

When $p$ is odd, in the decomposition of $\Omega P^{2 n+1}\left(p^{r}\right)$, constructed in [5], the most interesting factor is the so-called "bottom piece" $T$, the one whose homology contains the homology of $P^{2 n}\left(p^{r}\right)$. In [5] and [7], Cohen, Moore and Neisendorfer construct a homotopy fibration $G \longrightarrow T \longrightarrow \Omega S^{2 n+1}$, where $G$ is an $H$-space. They show that $G$ decomposes up to homotopy equivalence as a product $G \simeq S^{2 n-1} \times Z$ for some $Z$, thereby producing the map $\pi: \Omega^{2} S^{2 n+1} \longrightarrow S^{2 n-1}$ referred to above by composing the connecting map of the fibration with the projection to $S^{2 n-1}$. Things are fundamentally different for the prime 2 for generic values of $n$, since, according to Adams' solution of the Hopf invariant 1 problem [1], $S^{2 n-1}$ is almost never an $H$-space so a retraction of this sort will not be possible.

In this paper we find, in a theoretical sense, the bottom piece $T\left(P^{2 n}(2)\right)$ in the decomposition of $\Omega P^{2 n+1}(2)$ into indecomposable factors. For reasons mentioned above, it is not going to be possible to replicate the procedure of [4] and [7] at the prime 2. To this point, our decomposition has not led to a homotopy exponent for the space $P^{2 n+1}(2)$ or to an improved bound for the exponent of the 2 -torsion of $\pi_{*}\left(S^{2 n+1}\right)$. 
What we mean when we say that we have found $T\left(P^{2 n}(2)\right)$ in a theoretical sense is that we have reduced its computation to algebra. Unfortunately this algebra involves difficult unsolved questions concerning the mod 2 representation theory of the symmetric group. In particular, to determine the Euler-Poincaré series of $T$ we would need to be able to compute the largest projective submodule of the important $\mathbb{F}_{2}\left[S_{n}\right]$ module known as $\operatorname{Lie}(n)$, where $\mathbb{F}_{2}\left[S_{n}\right]$ is the group ring of the symmetric group $S_{n}$. Representation theory, which has good methods for solving such problems in characteristic 0 , does not at the moment have sufficient techniques for the modular (nonzero characteristic) case.

Our method for understanding $T\left(P^{2 n}(2)\right)$ is to show it equals the space $A^{\min }\left(P^{2 n}(2)\right)$ constructed in Selick and Wu's earlier paper [11]. In that paper, the last two authors described the finest natural decomposition of $\Omega \Sigma X$, where $X$ is a $p$-torsion suspension. (The results were later extended to $p$-local suspensions and to $p$-local coassociative co- $H$ spaces in [13] and [9].) In particular, Selick and Wu constructed natural functors $A^{\min }$ ( ) and $B^{\max }$ ( ) such that $\Omega \Sigma X \simeq A^{\min }(X) \times B^{\max }(X)$ where $H_{*}\left(A^{\min }(X)\right)$ contains $H_{*}(X)$ and is indecomposable in the sense that there is no natural retract of $A^{\mathrm{min}}$ ( ). While $A^{\mathrm{min}}(X)$ has no functorial decomposition it is possible, and often happens, that it might be decomposable for any particular space $X$. Indeed, as shown in [11] in the case where $p>2$ and $X=P^{2 n}(p)$, the space $T$ of Cohen, Moore and Neisendorfer does not equal $A^{\min }\left(P^{2 n}(p)\right)$ but is a proper retract. The main result of this paper shows, however, that $A^{\min }\left(P^{2 n}(2)\right)$ is indecomposable (up to homotopy) and so it is the bottom factor in the decomposition of $\Omega P^{2 n+1}(2)$.

Intuitively, the reason that $A^{\min }\left(P^{2 n}(2)\right)$ is indecomposable (up to homotopy) and $A^{\min }\left(P^{2 n}(p)\right)$ is not for $p>2$ can be traced to the fact that the coproduct of $\mathrm{Sq}^{2}$ contains $\mathrm{Sq}^{1} \otimes \mathrm{Sq}^{1}$ whereas we do not find $\beta \otimes \beta$ (where $\beta$ is the Bockstein) in coproducts at odd primes. This results in the wedge summands of the $n$-fold selfsmash products $\left(P^{2 n}(2)\right)^{(n)}$ being more tightly held together with Steenrod operations and thus not decomposing into a wedge of Moore spaces, as happens at odd primes. From a more technical point of view, the key ingredient in the proof is Lemma 2.1 which shows that Steenrod decompositions of the $n$-fold tensor product $V^{\otimes n}$, where $V=\bar{H}_{*}\left(\left(P^{2 n}(2)\right)\right.$, are the same as natural decompositions of $V^{\otimes n}$. This result depends heavily on the functorial decompositions of self-smash products developed by Selick and $\mathrm{Wu}$ in [12]. This is where the interplay with the mod 2 representation theory of the symmetric groups comes into consideration. Our result is obtained by examining the list of functorial indecomposable summands of $V^{\otimes n}$ given in [12].

Acknowledgements The authors are grateful for support from Engineering and Physical Sciences Research Council (EPSRC) of the UK, National Sciences Engineering Research Council of Canada and National University of Singapore. 


\section{Equality of Steenrod and functorial decompositions of $V^{\otimes n}$}

Let $V$ be the graded vector space $\langle u, v\rangle$. Let $\mathcal{B}$ denote the hyperalgebra $\mathcal{U}^{V}$ where $\mathcal{U}$ is as in [12]. Let $\mathcal{A}$ denote the Steenrod algebra.

Lemma 2.1 $\mathcal{A}$-module summands of $\bigoplus_{k} V^{\otimes n}$ are $\mathcal{B}$-module summands of $\bigoplus_{k} V^{\otimes n}$. Furthermore, such summands are restrictions to 2-variables of some $\mathcal{U}^{W}$-summand of $\bigoplus_{k} W^{\otimes_{n}}$ for $|W|=n$ or equivalently come from some idempotent in $\mathbf{k}\left[S_{n}\right]$.

Proof Any indecomposable summand of $\bigoplus_{k} V^{\otimes n}$ is a summand of $V^{\otimes n}$ by the unique decomposition of $\bigoplus_{k} V^{\otimes n}$ into indecomposable summands. $V^{\otimes n}$ is self-dual as an $\mathcal{A}$-module. Therefore dualizing the decomposition of $V^{\otimes n}$ in [12, Theorem 1.2] gives another decomposition as $\mathcal{A}$-modules. By inspection of the list of summands in [12, Theorem 1.2], we see that each indecomposable has a different top degree. Therefore in the correspondence between the pieces of these decompositions, each factor corresponds to itself. In other words, each factor is self-dual. By [12, middle of page 444] any self-dual $\mathcal{A}$-module has a canonical extension to a $\mathcal{B}$ module structure. Therefore the $\mathcal{A}$-module structure on each factor has a canonical extension to a $\mathcal{B}-$ module structure and so $\mathcal{A}$-module summands are $\mathcal{B}$-modules summands. The second statement is equivalent to [12, Theorem 5.8].

\section{Proof of the main theorem}

Before proving the main theorem, we recall several known facts mostly proven in [11].

Proposition 3.1 Let $B$ be a sub-Hopf algebra of $T(V)$. Then $B=T(S)$ for some $S \subset P T(V)$.

Lemma 3.2 Let $B$ be an $\mathcal{A}$-coalgebra retract of $T(W)$. Let $A=\mathbf{k} \square_{B} T(W)$. Then for any tensor length $m$ within $T(W)$, there is a split short exact sequence of $\mathcal{A}_{-}$ modules

$$
0 \longrightarrow Q B_{m} \longrightarrow A_{m-1} \otimes W \longrightarrow A_{m} \longrightarrow 0 \text {. }
$$

Corollary 3.3 Let $B$ be an $\mathcal{A}$-coalgebra retract of $T(W)$. Then for any $m, Q B_{m}$ is an $\mathcal{A}$-module retract of $W^{\otimes m}$.

Recall from [11] that $B^{\max }(V)$ is a functorial graded coalgebra retract of $T(V)$ and that

$$
B_{n}^{\max }(V)=P\left(B_{n}^{\max }(V)\right) \subseteq P\left(T_{n}(V)\right) .
$$


Let $L_{n}^{\max }(V)$ denote $B_{n}^{\max }(V)$ for $n>1$.

Theorem 3.4 Let $Z(W)$ be a natural submodule of $P_{m} T(W)$ which is a natural retract of $W^{\otimes m}$. Then $Z(W) \subset L_{m}^{\max }(W)$.

Theorem 3.5 $A^{\min }(V)$ is indecomposable as a coalgebra over $\mathcal{A}$.

Proof Recall that $V=\langle u, v\rangle$. Let $A^{\min , \mathcal{A}}(V)$ be the minimum coalgebra retract of $A^{\min }(V)$ over $\mathcal{A}$, with $\mathcal{A}$-module coalgebra maps $s: A^{\min , \mathcal{A}}(V) \longrightarrow T(V)$ and $r: T(V) \longrightarrow A^{\min , \mathcal{A}}(V)$ such that $r s=1_{A^{\min , \mathcal{A}}(V)}$. Let $p: T(V) \longrightarrow A^{\min , \mathcal{A}}(V)$ be the map defined as in [11, page 32]. Then $p$ is a morphism of coalgebras over $\mathcal{A}$. Set $B^{\max , \mathcal{A}}(V):=\mathbf{k} \square_{A^{\min , \mathcal{A}}(V)} T(V)$. By minimality of $A^{\min , \mathcal{A}}(V)$, ps is an isomorphism. Therefore the proof of $[11$, Proposition 6.1, (4) and (5)] goes through to show that $B^{\max , \mathcal{A}}(V)$ is a sub Hopf algebra of $T(V)$ over $\mathcal{A}$ and so $B^{\max , \mathcal{A}}(V)$ inherits a grading from the tensor length grading on $T(V)$. Similarly, if $C=\mathbf{k} \square_{A^{\min , \mathcal{A}}(V)} A^{\min }(V) \cong$ $\mathbf{k} \square_{B^{\max }(V)} B^{\max , \mathcal{A}}(V)$ then $C$ is a bigraded sub Hopf algebra of $B^{\max , \mathcal{A}}(V)$ and $A^{\min }(V) \cong A^{\min , \mathcal{A}}(V) \otimes C$. We show that $C=\mathbf{k}$ so that $A^{\min }(V)=A^{\min , \mathcal{A}}(V)$.

If $C \neq \mathbf{k}$, let $n$ be the least nonvanishing tensor length degree of $C$. That is, if $C_{k}$ is the submodule of $C$ of tensor length $k$ we assume that $C_{k}=0$ for $k<n$. Then $C_{n}$ is a $\mathcal{A}$-module summand of $V^{\otimes n}$. Furthermore $C_{n}$ is contained in the primitives $P_{n} C$. Therefore by Lemma 2.1, $P_{n} C=C_{n}$ is an $\mathcal{B}$-module summand of $V^{\otimes n}$.

Let $Z=C_{n}$. By Corollary 3.3, $Z$ is an $\mathcal{A}$-module retract of $V^{\otimes n}$. By Lemma 2.1, there exists a functor $Z()$ such that $Z=Z(V)$. Equivalently, there exists an idempotent $e \in \mathbf{k}\left(S_{n}\right)$, such that $Z(W)=e W^{\otimes n}$ and in particular $Z=e V^{\otimes n}$. Write $e=\sum n_{i} e^{\lambda_{i}}$, where $e^{\lambda_{i}}$ is the idempotent for $P^{\lambda_{i}}$. Since we are dealing with a Moore space the dimension is 2 thus discarding those $\lambda_{i}$ with $\left|\lambda_{i}\right|>2$ (for more details see Selick and $\mathrm{Wu}$ [10]), we obtain a new $e$ (without changing $e V^{\otimes n}$ ) in which no partitions of length greater than 2 occur. We know that $Z \subset P_{n} T(V)$. Since $e$ has no partitions of length greater then $2, Z \subset L_{n} T(V)$ if and only if $Z() \subset L_{n} T()$.

Suppose $Z \nsubseteq L_{n} T V$. Let $T V=\otimes A_{i}$ be the minimal coalgebra decomposition of $T(V)$. Since $Z \subset P_{n} T V, Z \subset \oplus A_{i}$. Since the only factor in the corresponding decomposition of $V^{\otimes n}$ which consists entirely of primitives is $L_{n}^{\max }$, we conclude that $Z \subset L_{n}^{\max }$. Therefore there is copy of $B^{\max }$ which contains $Z$.

Since $A^{\min , \mathcal{A}}$ is a coalgebra retract of $A^{\min }(V)$, there is a choice of $B^{\max , \mathcal{A}}(V)$, as a sub-Hopf algebra of $T(V)$, which contains the sub-Hopf algebra $B^{\max }(V)$. We have 
a commutative diagram

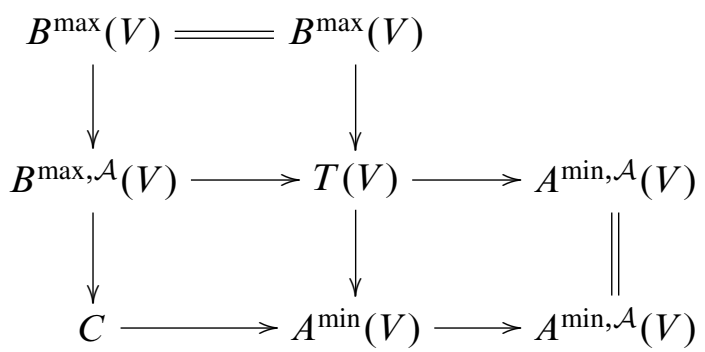

where $A^{\min }(V) \cong C \otimes A^{\min , \mathcal{A}}(V)$ as the lower left square commutes because the upper left square commutes. Thus the composite $B^{\max , \mathcal{A}}(V) \longrightarrow T(V) \longrightarrow A^{\min }(V)$ factors through $C=\mathbf{k} \square_{B^{\max }(V)} B^{\max , \mathcal{A}}(V)$.

The $B^{\max }(V)$ and $B^{\max , \mathcal{A}}$ are given by taking coalgebra kernel. Now, as $Z=C_{n} \subseteq$ $B^{\max }(V)$, the diagram implies that $C_{n}$ maps trivially to $C$, which can only occur if $C_{n}=\mathbf{k}$, and this contradicts the assumption that $C \neq \mathbf{k}$.

Corollary 3.6 $A^{\min }\left(P^{2 n}(2)\right)$ is indecomposable, up to homotopy. In particular it equals the indecomposable factor whose homology contains $H_{*}\left(P^{2 n}(2)\right)$ in the homotopy decomposition of $\Omega P^{2 n+1}(2)$.

\section{References}

[1] J F Adams, On the non-existence of elements of Hopf invariant one, Ann. of Math. (2) 72 (1960) 20-104 MR0141119

[2] F R Cohen, Applications of loop spaces to some problems in topology, from: "Advances in homotopy theory (Cortona, 1988)", London Math. Soc. Lecture Note Ser. 139, Cambridge Univ. Press (1989) 11-20 MR1055864

[3] F R Cohen, J C Moore, J A Neisendorfer, The double suspension and exponents of the homotopy groups of spheres, Ann. of Math. (2) 110 (1979) 549-565 MR554384

[4] F R Cohen, J C Moore, J A Neisendorfer, Torsion in homotopy groups, Ann. of Math. (2) 109 (1979) 121-168 MR519355

[5] F R Cohen, J C Moore, JA Neisendorfer, Exponents in homotopy theory, from: "Algebraic topology and algebraic $K$-theory (Princeton, N.J., 1983)", Ann. of Math. Stud. 113, Princeton Univ. Press (1987) 3-34 MR921471

[6] I M James, Reduced product spaces, Ann. of Math. (2) 62 (1955) 170-197 MR0073181

[7] J A Neisendorfer, 3-primary exponents, Math. Proc. Cambridge Philos. Soc. 90 (1981) 63-83 MR611286 
[8] P Selick, 2-primary exponents for the homotopy groups of spheres, Topology 23 (1984) 97-99 MR721456

[9] P Selick, S Theriault, J Wu, Functorial decompositions of looped coassociative co- $H$ spaces, Canad. J. Math. 58 (2006) 877-896 MR2245278

[10] P Selick, J Wu, Some calculations of $\operatorname{Lie}(n)^{\max }$ for low $n$, to appear in Journal of Pure and Applied Algebra

[11] P Selick, J Wu, On natural coalgebra decompositions of tensor algebras and loop suspensions, Mem. Amer. Math. Soc. 148 (2000) viii+109 MR1706247

[12] P Selick, J Wu, On functorial decompositions of self-smash products, Manuscripta Math. 111 (2003) 435-457 MR2002820

[13] P Selick, J Wu, The functor $A^{\text {min }}$ on p-local spaces, Math. Z. 253 (2006) 435-451 MR2221079

[14] S Theriault, Homotopy exponents of mod $2^{r}$ Moore spaces, to appear in J. Topol.

School of Mathematics, University of Manchester

M12 9PL, Manchester, United Kingdom

Department of Mathematics, University of Toronto

Toronto, Ontario M5S 3G3, Canada

Department of Mathematics, National University of Singapore

Singapore 119260, Singapore

Jelena.Grbic@manchester.ac.uk, selick@math.toronto.edu,

matwuj@nus. edu.sg

http://www . maths.manchester.ac.uk/ jelena,

http://www. math.toronto.edu/selick,

http://www.math.nus.edu.sg/ matwujie

Received: 26 December 2007 Revised: 28 March 2008

Algebraic 8 Geometric Topology, Volume 8 (2008) 